https://doi.org/10.52326/jes.utm.2021.28(1).02 UDC 621.565 .8

\title{
2D MODEL NUMERIC AMR, MODELLING AND SIMULATION OF MAGNETOCALORIC EFFECT
}

\author{
Dorin Botoc*, ORCID: 0000-0001-9561-9460 \\ "Gheorghe Asachi" Technical University of lasi, Boulevard Profesor Dimitrie Mangeron 65, Iași 700259, Romania \\ ${ }^{*}$ Corresponding author: Dorin Botoc, dorin.botoc@tuiasi.ro
}

Received: 01. 19. 2021

Accepted: 02. 22. 2021

\begin{abstract}
In this article, the study of gadolinium material is focused on determining the constants and properties of the material, the analysis of substructures and the orientation of dipole moments. Research into material aspects provides important information on atomic-level sub-phenomena in the field of the main magnetocaloric phenomena for the magnetic refrigeration regenerator (AMR). The experimental study is mainly based on testing the magneto-caloric material with different magnetization equipment, developed to measure more precise and fine details, magnetic refrigerator, AMR prototype development and the like. The method used uses differential equations. For both cases, where the loads are relatively simple and for more complex cases, the method can be easily applied.
\end{abstract}

Keywords: magnetic refrigeration, AMR, regenerable energy.

\section{Background and Introduction}

There is a considerable amount of literature available in which researchers have highlighted the benefits of the usage of magnetocaloric materials for the refrigeration processes and the potential of the magnetic materials has been demonstrated as an efficient energy saving materials. Gd et al studied in detail about the transduction of energy in ferromagnetic and ferroic materials, moreover the energy transduction in different types of materials such as piezo-electric, electromagnetic, and Magnetostrictive materials have been discussed in details and relevant advantages and disadvantages were also mentioned [1]. In Europe and America, huge amount of energy is wasted on the refrigeration and air conditioning, by adopting an energy efficient approach such as magnetocaloric refrigeration or magnetic refrigeration, a huge amount of energy can be saved [2]. The main reason for increased interest in the magnetic refrigeration is due to the environmentally friendly operation, highly energy efficient, and absence of requirement for the usage of harmful gases which cause ozone depletion and other effects such as greenhouse which contribute to the global warming.

The efforts are being made for the development of the refrigerator which can work at room temperature. There have been various successful attempts on the trial or experimental level, such as the individual products developed by General electric and Haier. The properties which are usable for the refrigeration effect are due to extraordinary 
response of these materials to external magnetic fields, the exhibition of such properties occur close to Curie temperature, it is the temperature at which the basic self-possessed magnetic properties are diminished, and the material temperature is dependent upon the application of the magnetic field. The effect mentioned previously has been in use since 1920 , for the examination of the magnetic structure and properties of iron and other related elements. The magnetocaloric effect was first discovered by the E. Warburg in 1881, who was a German physicist. The study tends to report the important developments and highlight the major breakthrough which have led to the acquirement of toady's knowledge and understanding about the magnetocaloric effect. Faraday's discovery that time variation of magnetic flux results in the induction of electric currents. Joule's understanding cleared the important concepts related to the electric currents and associated heat energy, and it was declared that heat energy released due to flow of electromagnetically induced current is equivalent to the heat energy produced due to the electric current produced by any other source, moreover, it was also inferred that rapid magnetization and demagnetization results in the heating of the magnetic material due to heat energy released as a result of current flow. Thomson inferred from the concepts of thermodynamics that temperature dependent property of magnetization of any material will represent or exhibit these properties related parameters in the form increase or decrease in temperature.

The accurate quantifiable measurement of magnetocalorific effect of iron was possible after a long time since the discovery of phenomena [3]. As there were issues related to high temperature which made the measurement of the related parameters challenging. The heat based electric motor was presented and produced by Tesla, Edison, and Stefan. Weiss and Langevin contributed separately the considerable knowledge and understanding about the temperature ranges, magnetization, and hysteresis. The initiation of the low temperature studies which had ultimately led to the realization and theoretical formulation of refrigeration was independently reported by Debye and Giauque that adiabtically demagnetized paramagnetic salts results in the attainment of low temperatures, Giauque and MacDougall showed the very low temperature achievements by the salts [4]. All the work reported above resulted in the development of foundation for the concepts building and actual understanding of magnetism, temperature, and material properties. Pecharsky et al. reported that the magnetic dipole moments of $\mathrm{Gd}$ can be put in order at room temperature $(294 \mathrm{~K})$, and the researchers highlighted that the continuous magnetic refrigeration was experimentally shown by the Collins and Zimmerman, they tested magnetic refrigerators operating at very low temperature ranges [5]. Zimm and DeGregoria explained the basic mechanism of Active magnetic regenerator cycle. Kitanovski et al. highlighted that the there is need and necessity to develop new thermodynamic cycles which can explain the magnetic refrigeration phenomena accurately and comprehensively and, the authors reported many publications about the Brayton and Ericsson's cycle associativity with the magnetic refrigeration, moreover, active magnetic regenerators with various thermodynamic cycles was analyzed, and numerical simulation of AMR was also performed using finite element method. The AMR operating on the Brayton cycle resulted in the production of highest cooling power capability, while AMR operating on the Ericsson cycle is the most efficient one [6], they also mathematically explained the numerical aspects of AMR simulation. Wolf et al provided quantum-based explanation of the magnetocaloric effect and proposed the increment or increase in the change in the entropy with respect to change in the magnetic field close to magnetically achieved 
quantum-critical point by the accurate and precise measurement of calorimetric effect [7]. Noume et al. used comsol of simulation of magnetocaloric effect for the designing of the magnetic regeneration cycle for the electric vehicles. As the electric vehicle operates on the battery, therefore, the energy would be derived from the battery, in the simulation performed, the fluid flow (mainly laminar), heat transfer in solids and fluids was used, and the velocity, temperature, and heat transfer coefficient was mainly studied [8].

\section{Boundary condition, mesh}

The primary equation which is used for the fluid flow is the Navier-Stokes equation, moreover, energy equation along with the heat transfer equations were mainly used [9]. Gobi and Sahu used COMSOL to perform the exploratory study of the magnetocaloric effect on three different materials, these materials were Gadolinium and two other different alloys for the evaluation of the final temperature of the magnetic material, the Gadolinium showed the adiabatic temperature difference of $12 \mathrm{~K}$ which was the highest among the studied material [10]. An application was developed having a graphical user interface (GUI), so that the user can input different variables, and visualize the contours of temperature and other variables. The mathematical based study is more equation oriented, in which mathematical techniques are employed to explain the physics of phenomena, and these study methods are valuable in terms of quantification of the variables and developing actual mathematical relations.

\section{Results and disscution}

The boundary condition of magnetic field is the Ampere's law which was applied on the regenerator, and the magnetization condition was defined. The magnetization model was applied, and the material was declared as solid, value of the magnetization defined was $222000 \mathrm{~A} / \mathrm{m}$ in $\mathrm{x}$ and $\mathrm{y}$ directions, and the values of electrical conductivities and relative permittivity were derived from the material. Magnetic insulation was also applied at the relevant boundaries.

The heat transfer in solids and fluids have boundary conditions of identification of the fluidic domain and solid domain, and the temperature was defined which was initial temperature of 293.15 Kelvin. The heat rate of 100 Watt was applied as the heat source at the regenerator. Similarly, the boundary conditions of the fluid ere applied defining inlet, outlet and wall. The normal inflow velocity of $5 \mathrm{~m} / \mathrm{s}$ was applied at the input, and the zero-velocity boundary condition was defined at the outlet. The proposed multiphysics option which the solver has provided consist of electromagnetic heating and non-isothermal flow conditions. The walls have been modeled with no slip boundary conditions.

The physics-controlled mesh was used for the simulation.

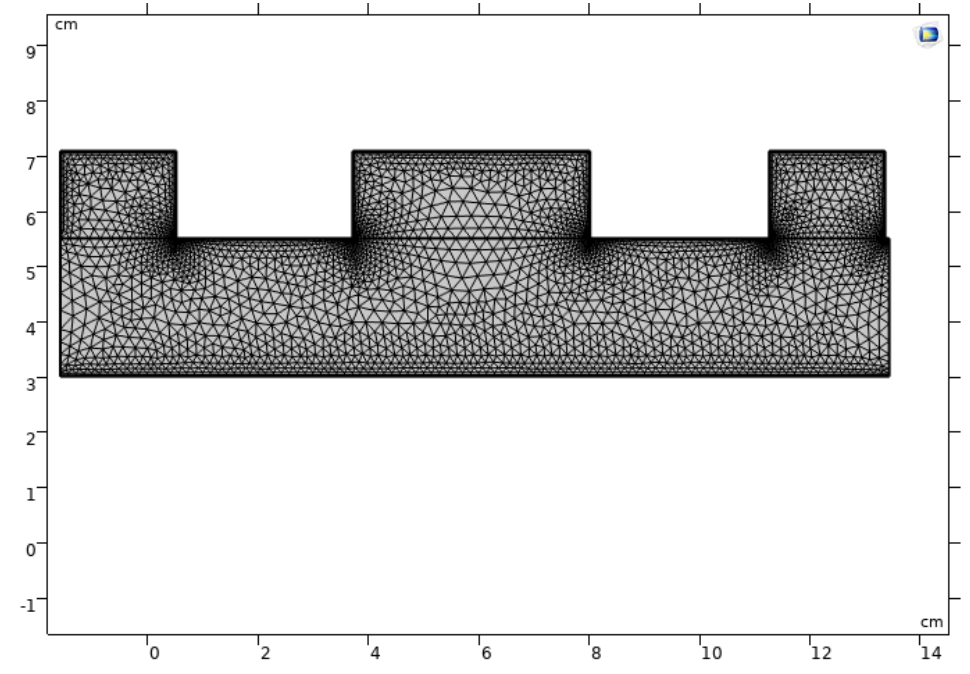

Figure 1. Comsol 2D model used for the simulation has been shown along with the mesh. 
Fig. 1 show the meshed domain, the mesh is very fine at the boundaries and edges.

The mesh is very fine at the boundaries and edges. The contributor of the mesh are magnetic fields, heat transfer in solids and fluids, laminar flow, electromagnetic heating, and non-isothermal flows.The stationary study was selected along with the PARSIDO solvers.

\section{Modelling and simulation}

The process modelling and simulation was performed in COMSOL multi-physics. The model was drawn in 2D and was inspired by the research performed by the Noume et al. 2D model was drawn in the Comsol model builder geometry tab. The model assumes to have symmetry.

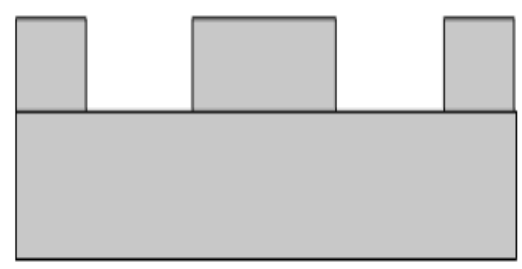

Figure 2. Geometrical representation of the model.

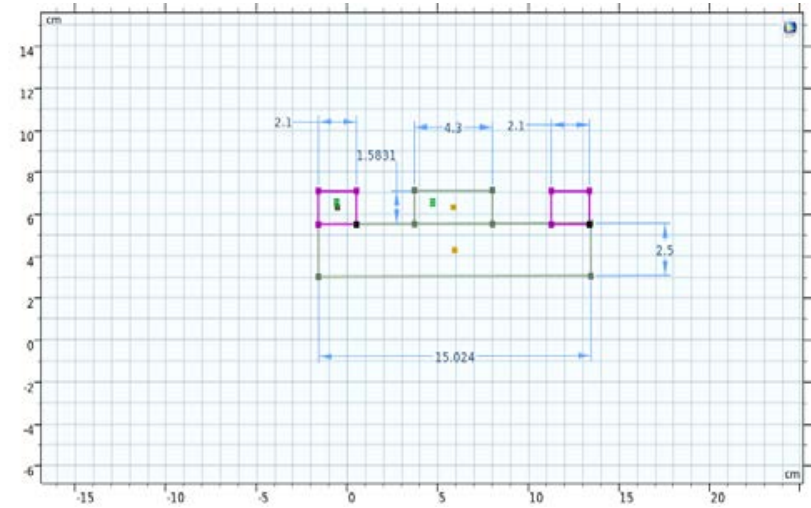

Figure 3. Comsol 2D AMR model used for the simulation of magnetocaloric effect along with the dimensions of various bodies used.

The length of the fluidic channel is $15.024 \mathrm{~cm}$, while the height of the fluidic channel was kept as $2.5 \mathrm{~cm}$. the magnetic regenerator has been placed at the center of the channel, and the width and height of the magnetic regenerator was kept as $4.3 \mathrm{~cm}$ and 1.5 $\mathrm{cm}$ respectively. The right-side square box was modeled as hot heat exchanger and the left side box was modelled as cold heat exchanger. The width and height of both the heat exchanger are 1.5 and $2.1 \mathrm{~cm}$ respectively. Figure 2 shows the complete dimensions of the model.

The materials were also selected according to the simulations performed by Noume et al.. The magnetic regenerator placed in the center was allotted the material Gadolinium, the fluid used in the simulation is water, and the plates used to model the heat exchanger is composed of copper. The material properties off copper used in the simulation along with comsol operators are shown below [11 - 13].

The physics selected for the simulations are the main determinants of the solution and boundary conditions. The models which were used in the simulation are, Magnetic fields, Heat transfer in solids, heat transfer in fluids, and Laminar flow for the fluidic solvers. All these physics have been coupled together to find a numerical solution of the magnetocaloric effect problem. COMSOL multi-physics excellently couples the physics to formulate a multi-physics problem, and the user friendly interface allows the user to add multiple studies within one window. The proposed multi-physics based on different physics selected are also shown in the multi-physics tab, and the option is provided to apply it on different domains and boundaries. All the previously defined steps allow the user to 
comprehensively define the problem [14 - 15]. Magnetic flux density norm along with temperature plot have been obtained. Fig.4 shows the magnetic flux density norm streamlines. Figure 5 also show the Magnetic flux density plot. The magnetization has produced the magnetic field.

The values of the temperature has been almost the same that were obtained by the Noume et al. in their simulations. The temperature rise due to magneto caloric effect is similar in magnitude that has been reported in the literature. It must be mentioned that the stationary study has been performed in order to simulate the process.

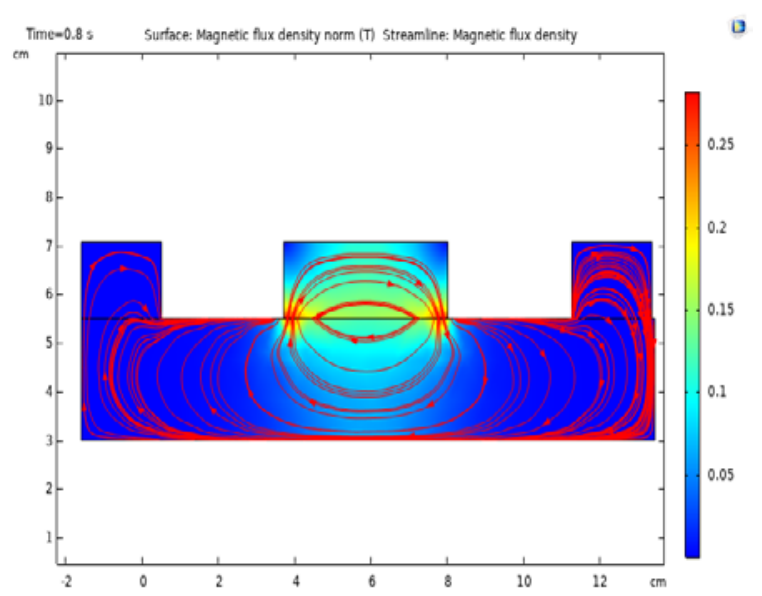

Figure 4. Magnetic flux density norm shown in the figure. The magnetization can be viewed from the streamlines.

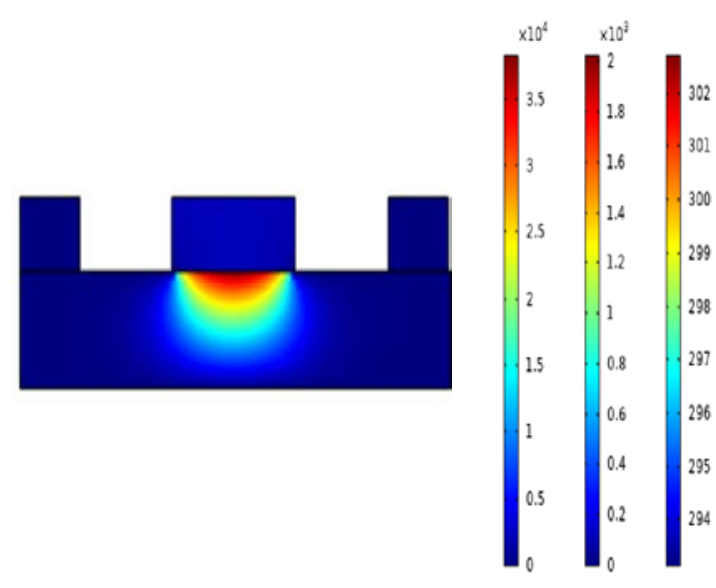

Figure 5. Temperature profile AMR.

Successful simulations have been performed by using the simplified model techniques and methods. For more realistic analysis transient approach should be adopted. The laminar flow approach has also been adopted in order to reduce the complexity of the solution procedure, otherwise the flow may be considered turbulent due to heat transfer and fluid flow in the current scenario. The material which was chosen for the study was widely used in the research of magnetocaloric materials.

There are other alloys which are members of lanthanide period have also been studied by the researchers in order to explore the potential for the refrigeration in the magnatocaloric materials. The tolerance set for the stationary solver was 0.001 default. MUMPS solver along with the PARSIDO solver was also used. The results have been evolved with the start of the flow. Figure 6 shows the contours of the magnetic flux density when the flow conditions were applied, for this case the magnetization was elevated to $450000 \mathrm{~A} / \mathrm{m}$.

Figure 7 shows the total heat flux magnitude. The heat energy dissipation can be viewed by examining the surface plot presented below.

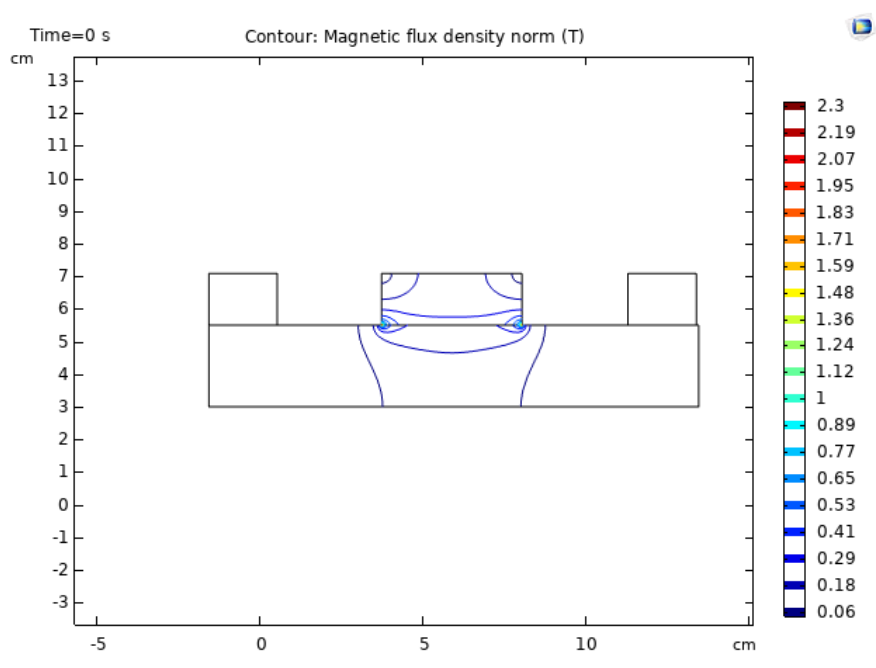

Figure 6. Magnetic flux density upon the application of the fluid flow conditions. 


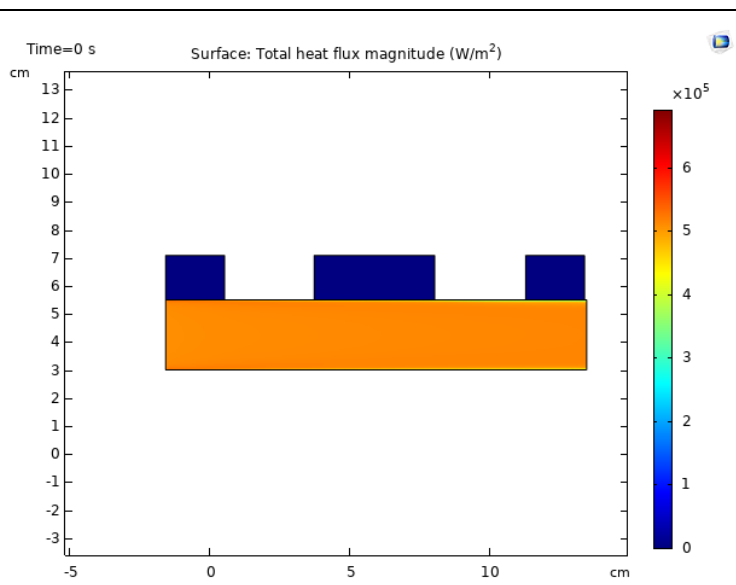

a)

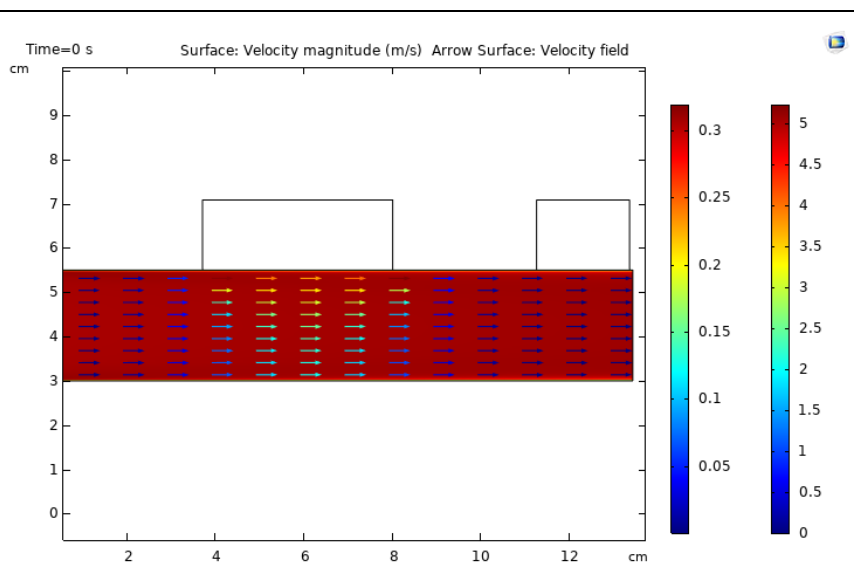

b)

Figure 7. a) Total heat flux magnitude plot, b) combined arrow and surface velocity plot just after the start of the flow.

The flow conditions which have been assumed for the analysis are very much simplified. The very simple yet comprehensive case studied combines the equations from the magnetic field, the heat transfer equations considering the effects of convection and conduction, and the flow equations which are continuity, momentum, and energy balance equations.

\section{Conclusions and perspectives}

In this study finite element analysis technique would be adopted in order to perform simulation material of magnetocaloric effect and parametric material study on the alloys. Finite element analysis is a numerical technique, in which the computational domain or body of interest is discretized into smaller discrete elements (mesh), and the relevant equations are solved for each mesh element or node. In this way the accurate and complete behavior of the object under defined loading conditions is attained. There are three methods normally used for the solution of a real world problem, these methods are, experimental, exact method (analytical) and numerical method. Experimental method provides accurate and actual solution of the problem, if the experiment is properly designed. However, for some cases it is difficult to design the experimental setup, moreover this method is expensive in the case when parameters have to be varied or the intension is to perform parametric study. The analytical method is based on the exact mathematical solution of the problem. This method uses the differential equations, for the simple cases in which loadings is simple, or for over simplified cases, the method can be applied easily.

\section{References}

1. A. Kitanovski, "Energy Applications of Magnetocaloric Materials", Advanced Energy Materials, vol. 10, no. 10, p. 1903741, 2020.

2. P. Shirron, "Applications of the magnetocaloric effect in single-stage, multi-stage and continuous adiabatic demagnetization refrigerators", Cryogenics, vol. 62, pp. 130-139, 2014

3. V. Franco, J. Blázquez, J. Ipus, J. Law, L. Moreno-Ramírez and A. Conde, "Magnetocaloric effect: From materials research to refrigeration devices", Progress in Materials Science, vol. 93, pp. 112-232, 2018

4. V. Pecharsky and K. Gschneidner, Jr., "Giant Magnetocaloric Effect in Gd5(Si2Ge2)", Physical Review Letters, vol. 78, no. 23, pp. 4494-4497, 1997

5. Eriksen D, Engelbrecht K, Bahl C, Bjørk R. “Active magnetic regenerator refrigeration with rotary multi-bed technology." Department of Energy Conversion and Storage, Technical University of Denmark, 2016. 146 p 
6. N. Mezaal, K. Osintsev and T. Zhirgalova, "Review of magnetic refrigeration system as alternative to conventional refrigeration system", IOP Conference Series: Earth and Environmental Science, vol. 87, p. 032024, 2017

7. S. Kulkarni, and A. Mashalkar, "Study of Magnetic Refrigeration", International Journal for Research in Engineering Application \& Management (IJREAM), no. -, 2018

8. W. Gao et al., "Energy transduction ferroic materials", Materials Today, vol. 21, no. 7, pp. 771-784, 2018

9. A. Smith, "Who discovered the magnetocaloric effect?", The European Physical Journal H, vol. 38, no. 4, pp. 507-517, 2013

10.V. Pecharsky and K. Gschneidner Jr, "Magnetocaloric effect and magnetic refrigeration", Journal of Magnetism and Magnetic Materials, vol. 200, no. 1-3, pp. 44-56, 1999

11. A. Kitanovski, U. Plaznik, J. Tušek and A. Poredoš, "New thermodynamic cycles for magnetic refrigeration", International Journal of Refrigeration, vol. 37, pp. 28-35, 2014

12.B. Wolf et al., "Magnetocaloric effect and magnetic cooling near a field-induced quantum-critical point", Proceedings of the National Academy of Sciences, vol. 108, no. 17, pp. 6862-6866, 2011

13.A. Noume, M. Risser and C. Vasile, "Modeling of a Magnetocaloric System for Electric Vehicles", in Comsol Conferrence, Rotterdam, 2013

14. S. Gombi and D. Sahu, "Exploration of selected room temperature magneto caloric materials using COMSOL multiphysics", IOP Conference Series: Materials Science and Engineering, vol. 577, p. 012160, 2019

15. C. Hsieh, Y. Su, C. Lee, P. Cheng and K. Leou, "Modeling of Graded Active Magnetic Regenerator for RoomTemperature, Energy-Efficient Refrigeration", IEEE Transactions on Magnetics, vol. 50, no. 1, pp. 1-4, 2014 